\title{
Perspectives of a myosin motor activator agent with increased selectivity
}

\author{
Péter Nánási Jr. ${ }^{1}$, István Komáromi ${ }^{2}$, János Almássy ${ }^{3} *$ \\ ${ }^{1}$ Department of Biophysics and Cell Biology, Faculty of Medicine, University of Debrecen, \\ Debrecen, Hungary \\ ${ }^{2}$ Division of Clinical Laboratory Science, Department of Laboratory Medicine, Faculty of \\ Medicine, University of Debrecen, Debrecen, Hungary \\ ${ }^{3}$ Department of Physiology, Faculty of Medicine, University of Debrecen, Debrecen, \\ Hungary \\ * Reprint requests to: János Almássy, Ph.D. \\ Department of Physiology, University of Debrecen, H-4012 Debrecen, Hungary \\ Phone/FAX: +36-52255575 / +36-52255116 \\ Email: almassy.janos@med.unideb.hu
}

Running title: Selectivity of myosin motor activators 


\begin{abstract}
Clinical treatment of heart failure is still not fully solved. A novel class of agents, the myosin motor activators, acts directly on cardiac myosin resulting in an increased force generation and prolongation of contraction. Omecamtiv mecarbil, the lead molecule of this group, is now in human 3 phase displaying promising clinical performance. However, omecamtiv mecarbil is not selective to myosin, since it readily binds to and activates cardiac ryanodine receptors (RyR-2), an effect that may cause complications is case of overdose. In this study, in silico analysis was performed to investigate the docking of omecamtiv mecarbil and other structural analogues to cardiac myosin heavy chain and RyR-2 in order to select the structure which has a higher selectivity to myosin over RyR-2. In silico docking studies revealed that omecamtiv mecarbil has comparable affinity to myosin and RyR-2: the respective $\mathrm{K}_{\mathrm{d}}$ values are 0.60 and $0.87 \mu \mathrm{M}$. Another compound CK-1032100 has much lower affinity to RyR-2 than omecamtiv mecarbil, while it still has a moderate affinity to myosin. It was concluded that further research starting from the chemical structure of CK-1032100 may result a better myosin activator burdened probably less by the RyR-2 binding side effect. It also is possible, however, that the selectivity of omecamtiv mecarbil to myosin over RyR-2 cannot be substantially improved, because similar moieties seem to be responsible for the high affinity to both myosin and RyR-2.
\end{abstract}

\title{
Key words:
}

Inotropic agents, Myosin activators, Omecamtiv mecarbil, Ryanodine receptor, Cytosolic $\mathrm{Ca}^{2+}$.

\section{Introduction}

Heart failure is a dominant factor of mortality in Europe and North America with progressively increasing prevalence. The failing heart is unable to produce the cardiac output required for sufficient perfusion of the body. In spite of the various inotropic strategies developed in the past, the clinical treatment of heart failure is still problematic. All the currently used positive inotropic agents, including the application of cardiac glycosides, $\beta$-adrenergic agonists, phosphodiesterase inhibitors and $\mathrm{Ca}^{2+}$ sensitizers, are burdened by more or less serious side-effects. Some of them, like cardiac glycosides, $\beta$ adrenergic agonists and phosphodiesterase inhibitors, are proarrhythmic due to the elevated cAMP level and the concomitant $\mathrm{Ca}^{2+}$ overload, which are known to cause early and late afterdepolarizations and increase cardiac oxygen consumption (January and Fozzard 1988; Priori and Corr 1990; January and Riddle 1990; Fozzard 1992; Tweedie et al. 2000; Ardehali et al. 2012). $\mathrm{Ca}^{2+}$ sensitizers are neither proarrhythmic, nor they increase cardiac oxygen demand, but may cause diastolic stiffness resulting in decreased ventricular filling (Lehmann et al. 2003; Endoh 2008).

Due to the above mentioned shortcomings a new generation of inotropes has recently been developed: the group of myosin motor activators, agents enhancing systolic force by direct modification of the actin-myosin interaction (Endoh 2008; Sun et al. 2008; Solaro 2009; Solaro 2010; Liu et al. 2015). Specifically, these compounds increase cardiac contractility by binding to the heavy chain of the cardiac myosin molecule, like omecamtiv mecarbil, the lead molecule of this group. The positive inotropic effect of omecamtiv mecarbil is based on its selective association to the S1 domain of the heavy 
chain of cardiac $\beta$-myosin at the site where the converter domain and the relay helix converge in the vicinity of the force-generating lever arm. The consecutive conformational change in the nucleotide-binding domain of myosin results in enhanced ATPase activity and also in robust alterations of the mechanical properties of myosin head (Malik and Morgan 2011). Due to the allosteric modulation of the nucleotidebinding domain, the inorganic phosphate production, which is the rate-limiting step of the actomyosin cycle, is accelerated by omecamtiv mecarbil. As a consequence, the transition rate between the weakly bound and the strongly bound configurations of the actin-myosin dimer also accelerates (Malik et al. 2011). The resultant higher number of forcegenerating cross-bridges increases the amplitude and lengthens the duration of contractions (Malik et al. 2011; Teerlink 2009; Mamidi et al. 2015; Winkelmann et al. 2015; Swenson et al. 2017).

Clinically, promising results have been obtained with omecamtiv mecarbil. Several studies, including phase-1, phase-2 and phase-3 ones, indicated that left ventricular systolic function was improved significantly by omecamtiv mecarbil in a dose-dependent manner. This included the elevation of stroke volume and left ventricular ejection time, associated with reduction of end-systolic and end-diastolic diameters of the ventricles (Cleland 2011; Teerlink et al. 2011; Teerlink et al. 2016a; Teerlink et al. 2016b; Galactic-HF Study). These beneficial effects were evident at therapeutically relevant concentrations (typically in the submicromolar range), however, higher, supratherapeutic concentrations (in the micromolar range) caused occasionally difficulties with ventricular filling and myocardial ischemia developing on the basis of the lengthened duration of systole on the expense of diastole (Cleland et al. 2011; Teerlink et al. 2011).

Since the compromised ventricular filling may also be related to altered function of the sarcoplasmic reticulum, the effect of omecamtiv mecarbil on canine RyR-2 was studied by monitoring the electrical activity of single RyR-2 channels incorporated into planar lipid bilayers (Nánási et al. 2017). Omecamtiv mecarbil significantly and reversibly increased the open probability and opening frequency of RyR-2 from the concentration of $1 \mu \mathrm{M}$. Accordingly, the mean closed time was reduced while the mean open time increased, indicating that increased open probability was mainly due to the increased frequency of opening induced by omecamtiv mecarbil. In addition, this RyR-2 activating effect of the agent was more prominent at low, close to diastolic intracellular $\mathrm{Ca}^{2+}$ levels. Pharmacological activation of the RyR-2 may be expected to enhance contractility - provided that this effect is more pronounced during systole than diastole. Experimentally the opposite was observed, i.e. the stimulatory effect of omecamtiv mecarbil on RyR-2 was the strongest at diastolic $\mathrm{Ca}^{2+}$ levels (Nánási et al. 2017). Indeed, diastolic shortening of single canine myocytes was more increased than systolic shortening in the presence of $10 \mu \mathrm{M}$ omecamtiv mecarbil, although this was not accompanied with the expected marked increases in cytosolic $\mathrm{Ca}^{2+}$ levels (Horváth et al. 2017).

Based on these results, the direct stimulatory action of omecamtiv mecarbil on RyR-2 has to be taken into account when discussing the mechanism of action and the potential effects or side effects of the compound. Or alternatively, the structure has to be modified in order to minimize its RyR-2 stimulating activity. This was attempted in the 
present study by performing in silico docking experiments with omecamtiv mecarbil and some related compounds on cardiac myosin and RyR-2.

\section{Mehods}

The results overviewed in the introduction made it clear that canine RyR-2 binds omecamtiv mecarbil with a reasonably high affinity. In order to find the binding site for omecamtiv mecarbil on the surface of RyR-2, the cryo-electron microscopy structure of the open-pore RyR-2 was downloaded from the PDB database; PDB ID: 5goa (Peng et al. 2016). Since RyR-2 is one of the largest ion channels, the downloaded structure was further simplified to be suitable for efficient docking experiments. In absence of information on how omecamtiv mecarbil can associate with RyR-2, first a "blind-docking" protocol was chosen, i.e. the whole protein surface was considered as a potential target for the drug. The rigid receptorflexible ligand docking model was performed by means of VINA (Trott and Olson 2010) using default parameters as implemented in the YASARA software (Krieger 2014).

\section{Results}

Excluding those poses which are situated inside the membrane and others which are on the surface of the membrane, the area containing the most probable binding sites for omecamtiv mecarbil are presented in Fig. 1.A, and a magnified view of the residues on this surface together with the binding pose of omecamtiv mecarbil are shown in Fig. 1.B. According to the results of docking, the role of residues S4154, S4156, Q4202, W4646, I4927 and N4928 have to be emphasized for the proposed binding pose for omecamtiv mecarbil. It is likely that hydrophobic interactions are intimately involved in binding of the agent. Further support is given to this binding pose by the good agreement between the presently estimated $\mathrm{K}_{\mathrm{d}}$ of 0.87 $\mu \mathrm{M}$ and the experimentally determined $\mathrm{K}_{\mathrm{d}}$ value of $1.6 \mu \mathrm{M}$ (Malik and Morgan 2011). Here is to be mentioned that our docking studies have some limitations, since the resolution of the RyR-2 protein structure is not optimal for high precision docking. In addition, the protein structure downloaded has many unresolved regions and the probably highly variable positions of these regions may influence the results obtained. However, bearing these limitations in mind, we believe that these docking studies can be regarded as a first attempt to shed light on the possible interactions between omecamtiv mecarbil and the cardiac ryanodine receptor.

One possible way to get rid of - or at least to minimize - the effect of myosin motor activators on RyR-2, is to find the optimal structure which is bound less to RyR-2, while is still bound relatively well to its therapeutic binding site, i.e. to the human beta-cardiac myosin motor domain receptor; PDB ID: 4pa0 (Winkelmann et al. 2015). Therefore omecamtiv mecarbil, together with its five ancestors shown in Fig. 2, were docked to both RyR-2 and the beta-cardiac myosin to determine their affinities simultaneously to the two binding sites. The first structure reported to enhance the ATPase activity of myosin was CK0156636. Water solubility was improved and protein binding was reduced by substitution of the nitrate group for fluorine in the successor, CK-1032100. The first agent found to increase fractional shortening of cardiac muscle was CK-1122534, but its selectivity was insufficient due to interactions with ATP-sensitive $\mathrm{K}^{+}$channels. In the next step CK-1213296 was developed. This compound failed to interfere with cardiac ion channels but suppressed the 
activity of CYP 1A2. All these unfavorable effects were eliminated from CK-1317138, while the optimal in vitro effects were obtained with omecamtiv mecarbil (CK-1827452), which was more effective than its ancestor, by one order of magnitude (Morgan et al. 2010; Teerlink et al. 2011; Aksel et al. 2015). Docking all these structures to RyR-2 and myosin was performed. In the case of myosin, the local docking/refinement protocol could be applied with default parameters, since here the binding site was well defined. The results are presented in Table 1. Only one compound, CK-1032100 was found to have a sufficiently low affinity to RyR-2 and a moderately high affinity to myosin - which is still too low for sufficient activation of the myosin heavy chain.

Since the two compounds, CK-0156636 and CK-1032100, both having unsubstituted nitrogen in the aromatic Ring 1 , had the lowest affinity (largest $\mathrm{K}_{\mathrm{d}}$ values) to RyR-2, our analysis suggests that substitution of nitrogen, and the consequent de-aromatization of Ring 1 , may be responsible for the increased affinity to RyR-2. This finding may initiate a new tailoring strategy for modification of Ring 1 in order to lowering the affinity of the compound to RyR-2.

\section{Discussion}

In this paper the mechanism of action of myosin motor activators, including the lead molecule of omecamtiv mecarbil, has been discussed in details. This new group of agents was designed to support the cardiac function of patients with heart failure by utilizing their unique ability to increase the number of force generating actomyosin cross bridges. The molecule is currently in human phase 3, its further development is apparently promising (Meijs et al. 2012; Garg and Frishman 2013; Liu et al. 2016; Moin et al. 2016). On the other hand, omecamtiv mecarbil is not so selective as believed earlier because it activates RyR-2 receptors as well (Nánási et al. 2017). In the present study an attempt was made to identify the underlying binding site using in silico analysis and a possible direction of modification of the chemical structure has been proposed in order to increase its selectivity to myosin. It must be emphasized that our results may be considered only as suggestions regarding the direction of possible chemical modifications because of the limitations mentioned before. An alternative possibility that omecamtiv mecarbil can not be made much more selective to myosin can not be ruled out either, since similar structures seem to be responsible for the high affinity to myosin as well as to RyR-2. In this case the RyR-2 activating effect of omecamtiv mecarbil has to be considered as an unavoidable side-effect of the drug. Further studies, based on the proposed outlines of the structure-activity relationship (e.g. development and probing compounds with aromatic Ring 1), are required to clarify this point.

Regarding the clinical application of omecamtiv mecarbil in the future, extra care has to be taken in cases of overdose since supratherapeutic concentrations of omecamtiv mecarbil may cause complications due to activation of cardiac ryanodine receptors. Although cytosolic $\mathrm{Ca}^{2+}$ concentrations were not increased significantly by omecamtiv mecarbil (only a small, non significant elevation in the amplitude of the $\mathrm{Ca}^{2+}$ transient could be observed, Horváth et al. 2017), this does not exclude the possibility of $\mathrm{Ca}^{2+}$ leak from the SR. $\mathrm{Ca}^{2+}$-sensitive fluorescent dyes monitor cytosolic $\mathrm{Ca}^{2+}$ mainly in the bulk phase, while the $\mathrm{Ca}^{2+}$ leak is directed into the fuzzy space, from where it can be eliminated rapidly. The enhancement of the main $\mathrm{Ca}^{2+}$-eliminating mechanism, the forward mode $\mathrm{Na}^{+} / \mathrm{Ca}^{2+}$ exchange, may generate 
an inward current which was shown to be proarrhythmic (January and Riddle 1990). In addition, the diastolic $\mathrm{Ca}^{2+}$ leak may also contribute to the development of diastolic stiffness combined with angina, observed in some patients with omecamtiv mecarbil overdose. It must be born in mind that all these complications are anticipated only in cases of overdose intoxication when the plasma concentration of omecamtiv mecarbil reaches highly supratherapeutic levels. 


\section{Acknowledgements}

This work was supported by a grant provided to JA from the National Research Development and Innovation Office (NKFIH PD112199). JA was also supported by the Janos Bolyai Research Scholarship of the Hungarian Academy of Sciences, Lajos Szodoray Scholarship of the University of Debrecen and by the Campus Hungary Mobility Program. Dr István Komáromi, the co-author responsible for in silico docking studies, has died in 2017.

\section{Conflict of interest}

The authors of this manuscript wish to declare no competing interest. 


\section{References}

Ardehali, H.; Sabbah, H.N.; Burke, M.A.; Sarma, S.; Liu, P.P.; Cleland, J.G.; Maggioni, A.; Fonarow, G.C.; Abel, E.D.; Campia, U.; Gheorghiade, M. 2012. Targeting myocardial substrate metabolism in heart failure: potential for new therapies. Eur. J. Heart. Fail. 14: 120-129.

Aksel, T.; Yu, E.C.; Sutton, S.; Ruppel, K.M.; Spudich, J.A. 2015. Ensemble force changes that result from human cardiac myosin mutations and a small-molecule effector. Cell. Rep. 11: 910-920.

Cleland, J.G.F.; Teerlink, J.R.; Senior, R.; Nifontov, E.M.; McMurray, J.J.V.; Lang, C.C.; Tsyrlin, V.A.; Greenberg, B.H.; Mayet, J.; Francis, D.P.; Shaburishvili, T.; Monaghan, M.; Saltzberg, M.; Neyses, L.; Wasserman, S.M.; Lee, J.H.; Saikali, K.G.; Clarke, C.P.; Goldman, J.H.; Wolff, A.A.; Malik, F.I. 2011. The effects of the cardiac myosin activator, omecamtiv mecarbil, on cardiac function in systolic heart failure: a double-blind, placebo-controlled, crossover, dose-ranging phase 2 trial. Lancet 378: 676-683.

Endoh, M. 2008. Cardiac $\mathrm{Ca}^{2+}$ signaling and $\mathrm{Ca}^{2+}$ sensitizers. Circ. J. 72: 1915-1925.

Fozzard, H.A. 1992. Afterdepolarizations and triggered activity. Basic Res. Cardiol. 87 Suppl 2: 105-113.

A double-blind, randomized, placebo-controlled, multicenter study to assess the efficacy and safety of omecamtiv mecarbil on mortality and morbidity in subjects with chronic heart failure with reduced ejection fraction (GALACTIC-HF) clinicaltrials.gov; NCT02929329.

Garg, V.; Frishman, W.H. 2013. A new approach to inotropic therapy in the treatment of heart failure: cardiac myosin activators in treatment of HF. Cardiol. Rev. 21: 155159.

Greenberg, B.H.; Chou, W.; Saikali, K.G.; Escandón, R.; Lee, J.H.; Chen, M.M.; Treshkur, T.; Megreladze, I.; Wasserman, S.M.; Eisenberg, P.; Malik, F.I.; Wolff, A.A.; Shaburishvili, T. 2015. Safety and tolerability of omecamtiv mecarbil during exercise in patients with ischemic cardiomyopathy and angina. JACC Heart. Fail. 3: 22-29.

Horváth, B.; Szentandrássy, N.; Veress, R.; Almássy, J.; Magyar, J.; Bányász, T.; Tóth, A.; Papp, Z.; Nánási, P.P. 2017. Frequency-dependent effects of omecamtiv mecarbil on cell shortening of isolated canine ventricular cardiomyocytes. Naunyn Schmiedeberg's Arch. Pharmacol. 390: 1239-1246.

January, C.T.; Fozzard, H.A. 1988. Delayed afterdepolarizations in heart muscle: mechanism and relevance. Pharmacol. Rev. 40: 219-227.

January, C.T.; Riddle, M.J. 1990. Early afterdepolarizations: Mechanism of induction and block; A role for L-type $\mathrm{Ca}^{2+}$ current. Circ. Res. 64: 977-990.

Krieger E. 2014. Yasara view - molecular graphics for all devices - from smartphones to workstations. www.yasara.org.

Lehmann, A.; Boldt, J.; Kirchner, J. 2003. The role of $\mathrm{Ca}^{++}$-sensitizers for the treatment of heart failure. Curr. Opin. Crit. Care 9: 337-344.

Liu, Y.; White, H.D.; Belknap, B.; Winkelmann, D.A.; Forgacs, E. 2015. Omecamtiv mecarbil modulates the kinetic and motile properties of porcine $\beta$-cardiac myosin. Biochemistry 54: 1963-1975. 
Liu, L.C.; Dorhout, B.; Van der Meer, P.; Teerlink, J.R.; Voors, A.A. 2016. Omecamtiv mecarbil: a new cardiac myosin activator for the treatment of heart failure. Expert. Opin. Invest. Drugs 25: 117-127.

Malik, F.I.; Morgan, B.P. 2011. Cardiac myosin activation part 1: From concept to clinic. J. Mol. Cell. Cardiol. 51: 454-461.

Malik, F.I., Hartman, J.J.; Elias, K.A.; Morgan, B.P.; Rodriguez, H.; Brejc, K.; Anderson, R.L.; Sueoka, S.H.; Lee, K.H.; Finer, J.T.; Sakowicz, R.; Baliga, R.; Cox, D.R.; Garard, M.; Godinez, G.; Kawas, R.; Kraynack, E.; Lenzi, D.; Lu, P.P.; Muci, A.; Niu, C.; Qian, X.; Pierce, D.W.; Pokrovskii, M.; Suehiro, I.; Sylvester, S.; Tochimoto, T.; Valdez, C.; Wang, W.; Katori, T.; Kass, D.A.; Shen, Y.T.; Vatner, S.F.; Morgans, D.J. 2011. Cardiac myosin activation: a potential therapeutic approach for systolic heart failure. Science 331: 1439-1443.

Mamidi, R.; Gresham, K.S.; Li, A.; dos Remedios, C.G.; Stelzer, J.E. 2015. Molecular effects of the myosin activator omecamtiv mecarbil on contractile properties of skinned myocardium lacking cardiac myosin binding protein-C. J. Mol. Cell. Cardiol. 85: 262-272.

Meijs, M.F.L.; Asselbergs, F.W.; Doevendans, P.A. 2012. Omecamtiv mecarbil: a promising new drug in systolic heart failure. Eur. J. Heart Fail. 14: 232-233.

Moin, D.S.; Sackheim, J.; Hamo, C.E.; Butler, J. 2016. Cardiac myosin activators in systolic heart failure: More friend than foe? Curr. Cardiol. Rep. 18: 100.

Morgan, B.P.; Muci, A.; Lu, P.-P.; Qian, X.; Tochimoto, T.; Smith, W.W.; Garard, M.; Kraynack, E.; Collibee, S.; Suehiro, I.; Tomasi, A.; Valdez, S.C.; Wang, W.; Jiang, H.; Hartman, J.; Rodriguez, H.M.; Kawas, R.; Sylvester, S.; Elias, K.A.; Godinez, G.; Lee, K.; Anderson, R.; Sueoka, S.; Xu, D.; Wang, Z.; Djordjevic, N.; Malik, F.I.; Morgans, D.J. 2010. Discovery of omecamtiv mecarbil the first, selective, small molecule activator of cardiac myosin. ACS Med. Chem. Lett. 7: 472-477.

Nánási, P.Jr.; Gaburjakova, M.; Gaburjakova, J.; Almássy, J. 2017. Omecamtiv mecarbil activates ryanodine receptors from canine cardiac but not skeletal muscle. Eur. J. Pharmacol. 809: 73-79.

Peng, W.; Shen, H.; Wu, J.; Guo, W.; Pan, X.; Wang, R.; Chen, S.R.W.; Yan N. 2016. Structural basis for the gating mechanism of the type 2 ryanodine receptor RyR2. Science 354: Issue 6310.

Priori, S.G.; Corr, P.B. 1990. Mechanisms underlying early and delayed afterdepolarizations induced by catecholamines. Am. J. Physiol. 258: H1796H1805.

Solaro, R.J. 2009. Maintaining cooperation among cardiac myofilaments proteins through thick and thin. J. Physiol. (Lond.) 587, 3.

Solaro, R.J. 2010. Sarcomere control mechanisms and the dynamics of the cardiac cycle. J. Biomed. Biotechnol. 8: Article ID 105648.

Sun, Y.B.; Lou, F.; Irving, M. 2008. Calcium- and myosin-dependent changes in troponin structure during activation of heart muscle. J. Physiol. (Lond.) 587: 155-163.

Swenson, A.M.; Tang, X.W.; Blair, C.A.; Fetrow, C.M.; Unrath, W.C.; Previs, M.J.; Campbell, K.S.; Yengo, C.M. 2017. Omecamtiv mecarbil enhances the duty ratio 
of human-cardiac myosin resulting in increased calcium sensitivity and slowed force development in cardiac muscle. J. Biol. Chem. 292: 3768-3778.

Teerlink, J.R. 2009. A novel approach to improve cardiac performance: cardiac myosin activators. Heart Fail. Rev. 14, 289-298.

Teerlink, J.R.; Clarke, C.P.; Saikali, K.G.; Lee, J.H.; Chen, M.M.; Escandon, R.D.; Elliott, L.; Bee, R.; Habibzadeh, M.R.; Goldman, J.H.; Schiller, N.B.; Malik, F.I.; Wolff, A.A. 2011. Dose-dependent augmentation of cardiac systolic function with the selective cardiac myosin activator, omecamtiv mecarbil: a first-in-man study. Lancet 378: 667-675.

Teerlink, J.R.; Felker, G.M.; McMurray, J.J.V.; Ponikowski, P.; Metra, M.; Filippatos, G.S.; Ezekowitz, J.A.; Dickstein, K.; Cleland, J.G.F.; Kim, J.B.; Lei, L.; Knusel, B.; Wolff, A.A.; Malik, F.I.; Wasserman, S.M.; on behalf of the ATOMIC-AHF Investigators. 2016a. Acute treatment with omecamtiv mecarbil to increase contractility in acute heart failure: the ATOMIC-AHF study. J. Am. Coll. Cardiol. 67: 1444-1455.

Teerlink, J.R.; Felker, G.M.; McMurray, J.J.; Solomon, S.D.; Adams, K.F. Jr; Cleland, J.G.; Ezekowitz, J.A.; Goudev, A.; Macdonald, P.; Metra, M.; Mitrovic, V.; Ponikowski, P.; Serpytis, P.; Spinar, J.; Tomcsányi, J.; Vandekerckhove, H.J.; Voors, A.A.; Monsalvo, M.L.; Johnston, J.; Malik, F.I.; Honarpour, N.; for the COSMIC-HF Investigators. 2016b. Chronic Oral Study of Myosin Activation to Increase Contractility in Heart Failure (COSMIC-HF): a phase 2, pharmacokinetic, randomised, placebo-controlled trial. Lancet 388: 2895-2903.

Trott, O.; Olson, A.J. 2010. AutoDock Vina: improving the speed and accuracy of docking with a new scoring function, efficient optimization, and multithreading. J. Comput. Chem. 31: 455-461.

Tweedie, D.; Harding, S.E.; MacLeod, K.T. 2000. Sarcoplasmic reticulum Ca content, sarcolemmal $\mathrm{Ca}$ influx and the genesis of arrhythmias in isolated guinea-pig cardiomyocytes. J. Mol. Cell. Cardiol. 32: 261-272.

Vu, T.; Ma, P.; Xiao, J.; Wang, Y.M.; Malik, F.I.; Chow, A.T. 2015. Population pharmacokinetic-pharmacodynamic modeling of omecamtiv mecarbil, a cardiac myosin activator, in healthy volunteers and patients with stable heart failure. $\mathrm{J}$. Clin. Pharmacol. 55: 1236-1247.

Winkelmann, D.A.; Forgacs, E.; Miller, M.T.; Stock, A.M. 2015. Structural basis for drug-induced allosteric changes to human b-cardiac myosin motor activity. Nature Com. 6: 7974. 


\section{Table 1.}

Affinity of omecamtiv mecarbil (OM) and its ancestors to the cardiac ryanodine receptor (RyR-2) and myosin heavy chain. Dissociation constants $\left(\mathrm{K}_{\mathrm{d}}\right)$ and binding energies were obtained by docking the compounds to RyR-2 and myosin.

\begin{tabular}{lllll} 
& \multicolumn{2}{c}{ RyR-2 } & \multicolumn{2}{c}{ Myosin } \\
& Binding & $\begin{array}{l}\mathrm{K}_{\mathrm{d}} \\
\text { energy } \\
(\mathrm{kcal} / \mathrm{mol})\end{array}$ & $\begin{array}{l}\text { Binding } \\
\text { energy } \\
(\mathrm{kcal} / \mathrm{mol})\end{array}$ & $\mathrm{K}_{\mathrm{d}}$ \\
CK-0156636 & 6.28 & 24.93 & 6.74 & 11.43 \\
CK-1032100 & 6.27 & 25.35 & 7.30 & 4.49 \\
CK-1122534 & 8.59 & 0.50 & 6.74 & 11.39 \\
CK-1213296 & 7.83 & 1.83 & 6.92 & 8.52 \\
CK-1317138 & 7.70 & 2.26 & 5.82 & 53.82 \\
CK-1827452 (OM) & 8.27 & 0.87 & 8.49 & 0.60
\end{tabular}




\section{Figure legends}

Fig. 1. A: The RyR-2 receptor model used for docking and the position of omecamtiv mecarbil on its surface. B: A close view of omecamtiv mecarbil in its most likely docked pose obtained from docking experiments. Residues likely involved in omecamtiv mecarbil binding are marked individually. The figures were prepared using the YASARA software.

Fig. 2. Chemical structure of omecamtiv mecarbil (methyl 4-[(2-fluoro-3-\{[N-(6methylpyridine-3-yl)carbamoyl]amino \} phenyl)methyl] piperazine-1-carboxylate), known also as CK-1827452, and its ancestors showing the steps of drug development. Structural modifications are indicated by circles. 

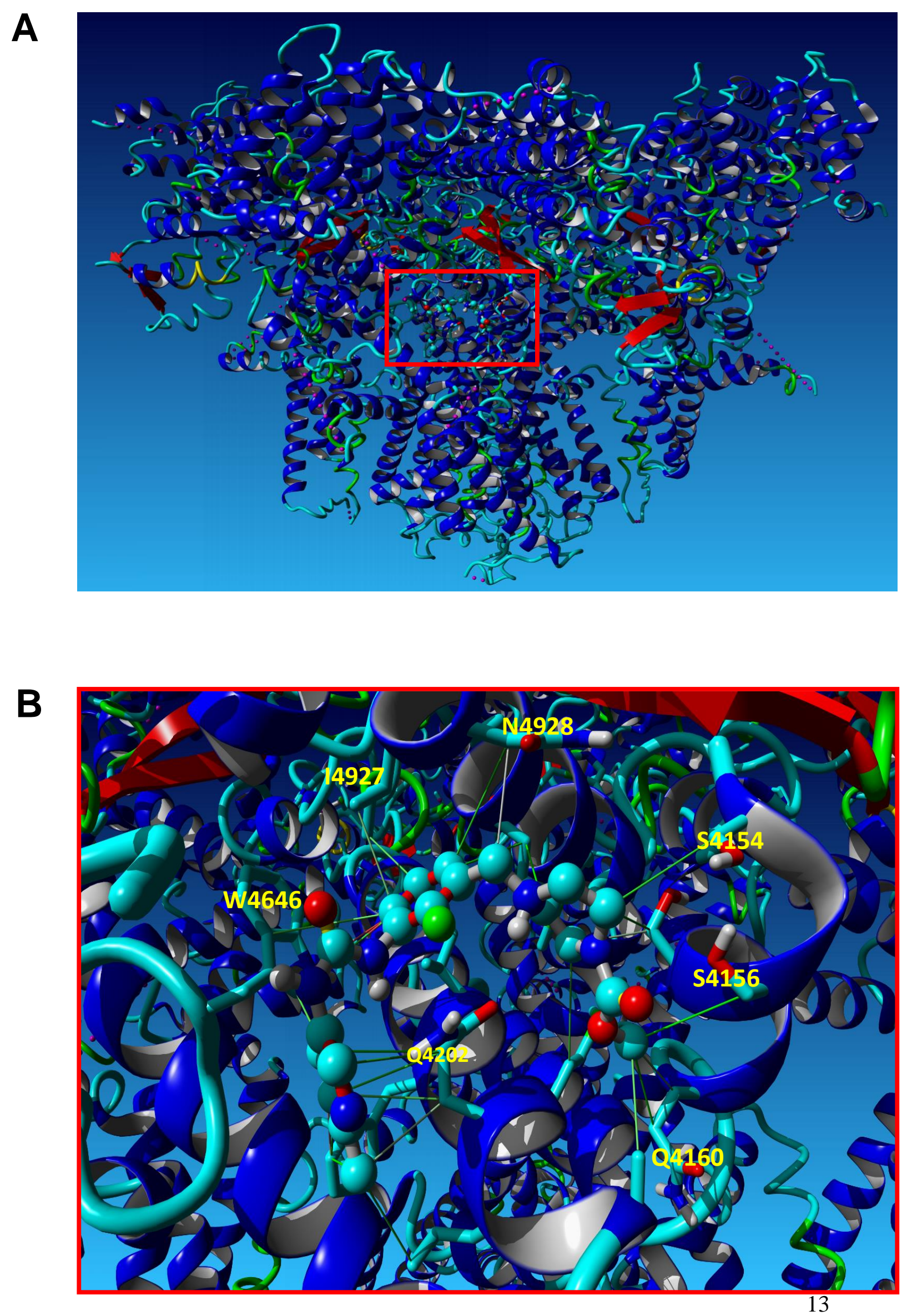

Fig. 1 


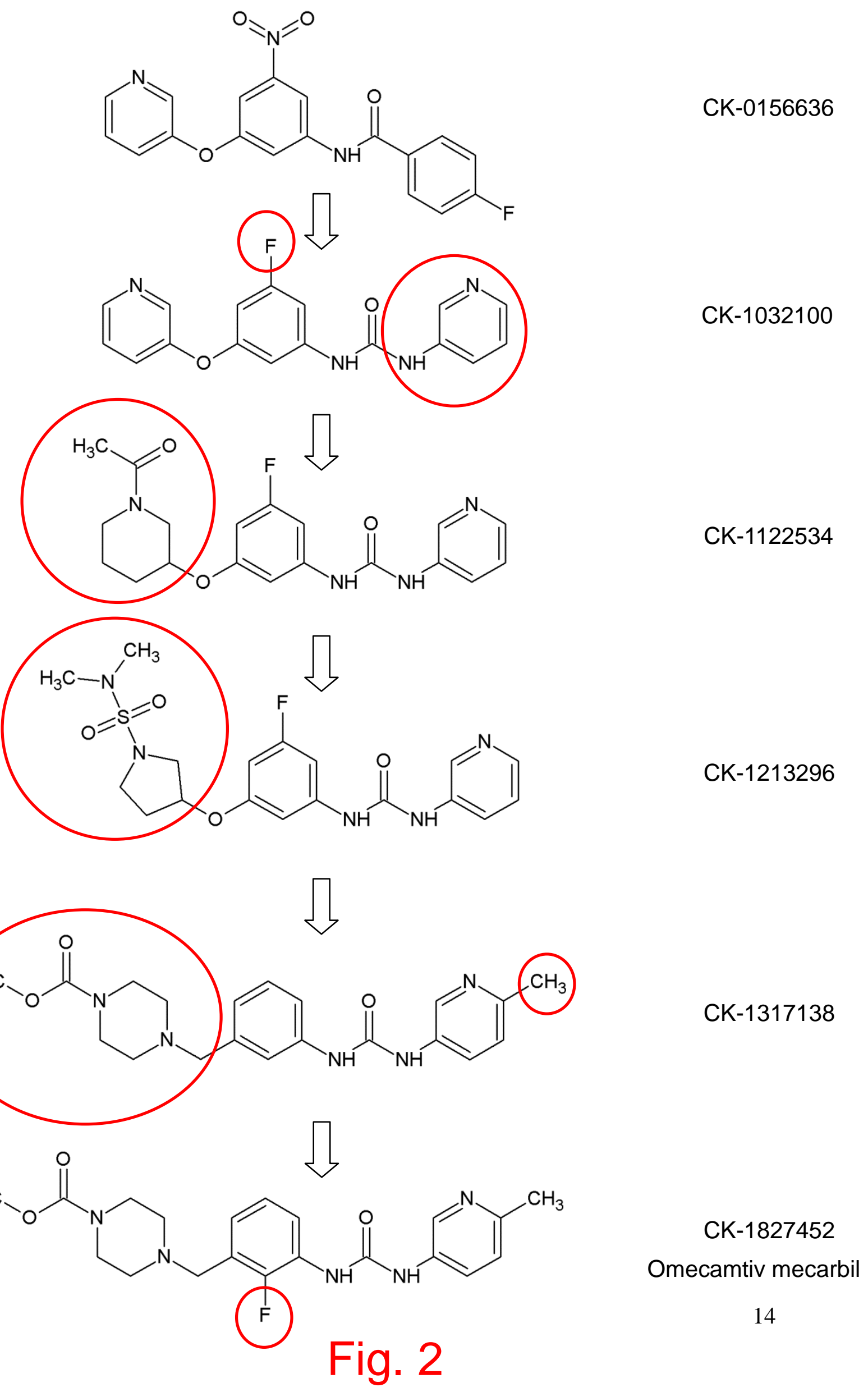

